Signal \& Image Processing : An International Journal(SIPIJ) Vol.1, No.2, December 2010

\title{
PERFORMANCE OF VARIOUS ORDER STATISTICS FILTERS IN IMPULSE AND MIXED NOISE REMOVAL FOR RS IMAGES
}

\author{
Mrs V.Radhika ${ }^{1} \&$ Dr G. Padmavathi ${ }^{2}$ \\ 1. Prof \&Head, Department of Computer Science, Sri Krishna Arts \& Science \\ College, Coimbatore-641 008. \\ (radhikavin73eyahoo.com) \\ 2. Prof \& Head, Department of Computer Science, Avinashilingam University \\ for Women, Coimbatore-641 043.
}

\begin{abstract}
Remote sensing images (ranges from satellite to seismic) are affected by number of noises like interference, impulse and speckle noises. Image denoising is one of the traditional problems in digital image processing, which plays vital role as a pre-processing step in number of image and video applications. Image denoising still remains a challenging research area for researchers because noise removal introduces artifacts and causes blurring of the images. This study is done with the intension of designing a best algorithm for impulsive noise reduction in an industrial environment. A review of the typical impulsive noise reduction systems which are based on order statistics are done and particularized for the described situation. Finally, computational aspects are analyzed in terms of PSNR values and some solutions are proposed.
\end{abstract}

\section{KEYWORDS}

DENOISING, INTERFERENCE NOISE, SPECKLE NOISE, IMPULSE NOISE, PSNR, REMOTE SENSING IMAGE.

\section{INTRODUCTION}

Remote sensing [5] usually refers to the instrument based technology of acquiring information about the earth's surface (land and ocean) and atmosphere, using sensors onboard airborne (aircraft, balloons) or space-borne (satellites, space shuttles) platforms. The electromagnetic radiation is normally used as an information carrier in RS. Remote sensing employs passive and/or active sensors. Passive sensors are those which sense natural radiations, either reflected or emitted from the earth. On the other hand, the sensors which produce their own electromagnetic radiation are called active sensors (e.g. LIDAR, RADAR). Remote sensing can also be broadly classified as optical and microwave. In optical remote sensing, sensors detect solar radiation in the visible, near-, middle- and thermal-infrared wavelength regions, reflected/scattered or emitted from the earth, forming images resembling photographs taken by a camera/sensor located high up in space. Different land cover features, such as water, soil, vegetation, cloud and snow reflect visible and infrared light in different ways.

Interpretation of optical images requires the knowledge of the spectral reflectance patterns of various materials (natural or man-made) covering the surface of the earth. Measurement of spatially organized (most commonly, geographically distributed) data/information on some property(ies) (spectral; spatial; physical) of an array of target points (pixels) within the sensed scene that correspond to features, objects, and materials, doing this by applying one or more recording devices not in physical, intimate contact with the item(s) under surveillance (thus at a 
Signal \& Image Processing : An International Journal(SIPIJ) Vol.1, No.2, December 2010

finite distance from the observed target, in which the spatial arrangement is preserved); techniques involve amassing knowledge pertinent to the sensed scene (target) by utilizing electromagnetic radiation, force fields, or acoustic energy sensed by recording cameras, radiometers and scanners, lasers, radio frequency receivers, radar systems, sonar, thermal devices, sound detectors, seismographs, magnetometers, gravimeters, scintillometers, and other instruments.

The output of a remote sensing system is usually an image representing the scene being observed. Many further steps of digital image processing and modelling are required in order to extract useful information from the image. This not only increases the sensor's sensitivity, but, unfortunately, also increases the noise or "'grain," thus, generating images that are contaminated with random noise effects. Noise can be defined as any disturbance that changes the original signal information. Image noise is a random, usually unwanted, variation in brightness or colour information in an image. Image data recorded by sensors on a satellite or aircraft contain errors related to geometry and brightness values of the pixels. These errors are corrected using suitable (filters) mathematical models, which are either definite or statistical models.

Noise can be summarized as the visible effects of an electronic error or interference in the final image from a digital camera or any remote sensor. Noise is a function of how well the image sensor and digital signal processing systems inside the digital camera are prone to and can cope with or remove these errors or interference. Noise significantly degrades the image quality and increases the difficulty in discriminating fine details in the image. It also complicates further image processing, such as image segmentation and edge detection. Images are more prone to stuck-pixel noise (impulse) and other interference noises. One of the most important families of nonlinear image filters is based on order statistics the widely used median filter is the best known filter of this family. Nonlinear filters based on order statistics have excellent robustness properties in the presence of impulsive noise. They tend to preserve edge information, which is very important to human perception. Their computation is relatively easy and fast compared with some linear filters. All these features make them very popular in the image processing community.

This paper evaluates three median based impulse noise removal algorithms for remote sensing images to handle impulse noise and also see the performance of the filters in mixed noise. These three Denoising algorithms are based on classification of detection schemes and implemented for benchmark remote sensing images. The simulations are done in matlab 7.1 version.

The organization of this paper is as follows: Section 2 describes related work Section 3 discusses three noise removal algorithms based on different schemes; Section 4 discusses the experimental setup and results Section 5 presents the conclusion.

\section{NOISE MODEL}

Filtering a digital image to attenuate noise while keeping the image details preserved is an essential part of image processing. There are many works on the restoration of images corrupted by impulse noise. Various filtering techniques have been proposed for removing impulse noise in the past and it is well-known that linear filters could produce serious image blurring. As a result, nonlinear filters have been widely exploited due to their much improved filtering performance, in terms of mixed noise attenuation and edge / details preservation. Nonlinear techniques are mainly based on median or its modifications [4], which are robust estimators, immune to high levels of impulse noise and Gaussian noise. The major drawback of such algorithms is uniform application of particular filter across the entire image. Thus, besides noisy pixels, undisturbed pixels are also modified. Performances of these filters are enhanced by introducing an impulse detector and space-variant filtering, [2]-[8]. In this concept, each pixel is analyzed in context of its neighbourhood, and decision is made whether the pixel is noise-free or 
Signal \& Image Processing : An International Journal(SIPIJ) Vol.1, No.2, December 2010

corrupted. Accordingly, it will be left unchanged, or replaced by the estimated value, respectively. Constructing an impulse detector requires a trade-off between opposing demands for noise suppression and detail preservation. Impulse noise removal algorithms are classified based on the characteristics of the filtering schemes[2] and one algorithm is described for each scheme.

\section{- Filtering without Detection}

In this type of filtering a window mask is moved across the observed image. The mask is usually of size $(2 N+1) 2$, where $N$ is a positive integer. Generally the centre element is the pixel of interest. When the mask is moved starting from the left top corner of the image to the rightbottom corner, it performs some arithmetical operations without discriminating any pixel.

\section{- Detection followed by Filtering}

This type of filtering involves two steps. In first step it identifies noisy pixels and in second step it filters those pixels. Here also a mask is moved across the image and some arithmetical operations is carried out to detect the noisy pixels. Then filtering operation is performed only on those pixels which are found to be noisy in the previous step, keeping the non-noisy intact.

\section{- Hybrid Filtering}

In this type of filtering schemes, two or more filters are suggested to filter a corrupted location. The decision to apply a particular filter is based on the noise level at the test pixel location or performance of the filter on a filtering mask.

\section{FILTERING TECHNIQUES}

\subsection{Median Filter (MF)}

Median[1] Filter (MF) is a simple and powerful non-linear filter, based order statistics and easy to implement method of smoothing images, i.e. reducing the amount of intensity variation between one pixel and the next. It is often used to reduce noise in images. The idea of median filtering is simply to replace each pixel value in an image with the median value of pixels in the window. If $x(i, j)$ and $y(i, j)$ be the input and output respectively, then the median filter is defined as follows $Y(i, j)=$ median $\{X(i-s, j-t) l(s, t) \in W\}$ Where $W$ is the window that is defined in terms of the image co-ordinates in the neighbourhood of the origin.

\section{Fig 1. Method for Median Filter}

\begin{tabular}{|l|l|l|l|l|l|l|l|}
\hline & & & & & & & \\
\hline & 10 & 5 & 20 & & & & \\
\hline & 14 & 80 & 11 & & & & \\
\hline & 8 & 3 & 22 & & & & \\
\hline & & & & & & & \\
\hline & \multicolumn{7}{|c|}{$3,5,8,10,11,14,20,22,80$} \\
\hline
\end{tabular}

median (central value 80 is replaced by 11)

\section{The algorithm for the median filter is as follows:}

Step 1: Select a two dimensional window $\mathrm{W}$ of size $3 \times 3$. Assume that the pixel being processed is $\mathrm{Cx}, \mathrm{y}$.

Step 2: Compute - Wmed the median of the pixel values in the window $\mathrm{W}$.

Step 3: Repleace Cx,y by wmed.

Step 4: Repeat Steps 1 to 3 until all the pixels in the entire image are processed. 
Signal \& Image Processing : An International Journal(SIPIJ) Vol.1, No.2, December 2010

\section{Advantage:}

1. Easy to implement.

2. Good in denoising different types of noises.

\section{Disadvantage:}

1. Application of median filtering requires some caution because MF tends to remove image details such as thin lines and corners while reducing noise.

2. Moreover, median filtering performance is unsatisfactory in the case of signal dependant noise. To overcome these difficulties different variations of median filters have been developed.

\subsection{Decision Based Algorithm}

This Srini-Ebenezer algorithm[4] is based on second scheme ie filtering after detection. This algorithm consists of two stages. The first stage is to classify the corrupted and uncorrupted pixels and in the second stage, regularization method is applied to the corrupted pixels to preserve edges and suppress noise. The regularization method is nothing but replacing the the corrupted pixel by either by median pixel or neighborhood pixel by using a fixed window size of $3 \times 3$ resulting in lower processing time and good edge preservation.

The algorithm for the decision based filter is as follows:

Step 1: Select a two dimensional window $W$ of size $3 \times 3$. Assume that the pixel being processed is $\mathrm{Cx}, \mathrm{y}$.

Step 2: Compute - Wmin, Wmed and Wmax - the minimum,median and maximum of the pixel values in the window $\mathrm{W}$ respectively.

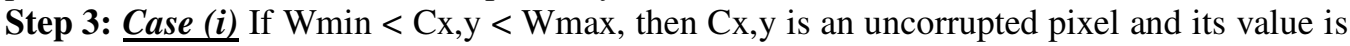
left unchanged.Otherwise $\mathrm{Cx}, \mathrm{y}$ is a noisy pixel.

Case (ii) If $\mathrm{Cx}, \mathrm{y}$ is a noisy pixel, it will be replaced by Wmed, the median value, only if Wmin $<$ Wmed $<$ Wmax.

Case (iii) If Wmin $<$ Wmed $<$ Wmax is not satisfied, Wmed itself is a noisy pixel value. In this case, $\mathrm{Cx}, \mathrm{y}$ will be replaced by the value of neighborhood pixels.

Step 4. Repeat Steps 1 to 3 until all the pixels in the entire image are processed.

\section{Advantage}

1. Less computations than many of the previous methods.

2. Better degree of denoising than many of median filter and its derivatives

\section{Disadvantage}

Although this method shows promising results there is a smooth transition between the pixels is lost leading to degradation in the visual quality of the image, since it only considers the left neighbourhood from the last processed value.

\subsection{Hybrid Filter[Hf]}

$\mathrm{HF}[3]$ is a class of non-linear filters which comes under the third scheme ie hybrid filtering. This model consists of two stages. The first stage consists of a fourth order PDE and the second stage is a relaxed median filter, which processes the output of fourth order PDE. The model enjoys the benefit of both nonlinear fourth order PDE and relaxed median filter. This method will not introduce any staircase effect and preserves fine details, sharp corners, curved structures and thin lines as in the previous methods.

Recently many authors proposed hybrid filters for image denoising. This paper focuses on the improved hybrid filter proposed by Jeny Rajan · K. Kannan · M.R. Kaimal[ 3]. to denoise low SNR molecular images. An alternative to median filter the relaxed median filter is used. This filter is obtained by relaxing the order statistic for pixel substitution. By using a relaxed median filter we can preserve more image details than the standard median filter. This method will not 
introduce any blocky effects in images (as can be seen in anisotropic median filter) and also preserve fine details, sharp corners and thin lines and curved structures to large extent.

Non-Linear $4^{\text {th }}$ order PDEs is an effective method of denoising. In this model L2 - curvature gradient flow method is used

$$
\frac{\partial u}{\partial x}=-\nabla^{2}\left[\left(\mathrm{c} \mid \nabla^{2} \mathrm{u}\right) \nabla^{2} \mathrm{u}\right]
$$

where $\nabla 2 u$ is the Laplacian of the image $u$. Since the Laplacian of an image at a pixel is zero if the image is planar in its neighborhood, the PDE attempt to remove noise and preserve edges by approximating an observed image with a piecewise planar image. $\mathrm{c}($.$) is the desirable diffusion$ coefficient diffuses more in smooth areas and less around less intensity transitions, so that small variations in image intensity such as noise and unwanted texture are smoothed and edges are preserved. Another objective for the selection of $c($.$) is to incur backward diffusion around$ intensity transitions so that edges are sharpened, and to assure forward diffusion in smooth areas for noise removal.

The discrete form of non-linear fourth order PDE described is

$$
U_{(i, j)}^{n+1}=U_{(i, j)}^{n}-\Delta t \nabla^{2} \mathrm{~g}(\mathrm{n}, \mathrm{n})
$$

Where,

$$
\begin{aligned}
& g_{i n}=g\left(\nabla^{2} U n_{i n}\right)
\end{aligned}
$$

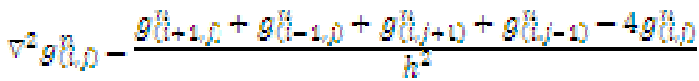

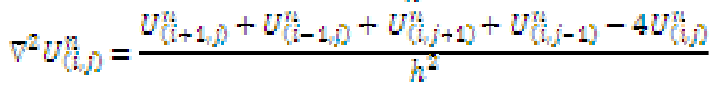

$\Delta \mathrm{t}$ is the time step size and $\mathrm{h}$ is the space grid size. Relaxed median filter can be used in with to remove large spike noise. The hybrid method is defined as follows,

$$
U_{i j}^{n+1}=R M_{\alpha \omega}\left(U_{i j}^{n}-\Delta t \nabla^{2} g_{i j}^{n}\right)
$$

Where RM is the relaxed median filter with lower bound $\alpha$ and upper band $\omega$. The output of the RM Yi,jcan be written as

$$
\begin{aligned}
& Y_{(i, j)}=R M_{\alpha} \omega\left\{W_{(i, j)}\right\} \\
& Y_{(i, D)}=R M_{G \omega}\left[W_{(i, j)}\right\}=\left\{\begin{array}{c}
X_{(D)} \quad \text { if } X_{(i, D}\left[\left[W_{(i, d}\right]_{\kappa}\left[W_{(i, D}\right]_{\omega}\right] \\
x \geq 0
\end{array}\right.
\end{aligned}
$$

\section{Advantage:}

Where $\left[\mathrm{W}_{(\mathrm{i}, \mathrm{j}}\right]_{\mathrm{m}}$ is the samples inside the window $\mathrm{W}_{(\mathrm{i}, \mathrm{j})}$

- When fourth order diffusion is applied to the images, the areas having small gradients are smoothed.

- The blocky effects can be avoided. The gradients generated by noise can be subsequently removed by a relaxed median filter without affecting the image structure.

- As iteration continues, the nonlinear PDE removes the low level noise and subsequently the relaxed median filter removes the impulsive noise spikes.

\section{Disadvantage:}

Only large number of iterations gives better results which results in more computations. 
Signal \& Image Processing : An International Journal(SIPIJ) Vol.1, No.2, December 2010

\section{EXPERIMENTAL SETUP \&RESULTS}

To test the performance of the above discussed Filtering algorithms, the following steps are executed.(Even they are analyzed for mixed noise also)

i) Uncorrupted RS image is taken as input.

ii) Impulse noises are added to the RS image.

iii) Third, the filtering algorithms are applied for reconstruction of RS images.

iv) Again add mixed noise to the uncorrupted RS image.

v) Then filtering algorithms are applied for reconstruction of RS images

[ vi) Wiener filter also applied to images to filter Gaussian noise and to compare

the values.]

To estimate the quality of reconstructed image, Mean Squared Error and Peak Signal to Noise Ratio are calculated for the original valuable and the reconstructed images. PSNR and MSE values are calculated using

$$
\text { PSNR }=10 \log _{10}\left[\frac{255^{2}}{M S E}\right]
$$

Where MSE represents the mean square error.

$$
M S E=\frac{1}{M N} \sum_{0, j}\left(x_{i, j}-y_{0, j}\right)^{2}
$$

The size of the image taken is concordaerial.png 3060 X 2036 X 3 pixels. First the image is converted to gray pixel, and then the algorithms are applied. The experiments are conducted using mat lab 7.1 version for mixed noise (impulse and Gaussian) and also for impulse. Next the different filtering algorithms are applied on noisy RS images. The parameters PSNR and MSE value are calculated for the noise free image and noisy image.

Table 1. PSNR Values for mixed noise

Table 2. PSNR Values for impulse noise

\begin{tabular}{|l|c|c|c|c|}
\hline \multirow{2}{*}{ Filter } & \multicolumn{2}{|c|}{ RVIN+Gaussian } & \multicolumn{2}{c|}{ SPN+Gaussian } \\
\cline { 2 - 5 } & $\mathbf{2 5 \%}$ & $\mathbf{5 0 \%}$ & $\mathbf{2 5 \%}$ & $\mathbf{5 0 \%}$ \\
\hline Median & 22.2745 & 19.4439 & 25.6925 & 14.615 \\
\hline HF & $\mathbf{2 7 . 2 1 1 1}$ & $\mathbf{2 1 . 5 7 2 8}$ & 25.6925 & 20.6299 \\
\hline DBAIN & 15.5283 & 12.5147 & $\mathbf{2 7 . 4 1 3}$ & $\mathbf{2 3 . 6 0 8 9}$ \\
\hline
\end{tabular}

\begin{tabular}{|l|c|c|c|c|}
\hline \multirow{2}{*}{ Filter } & \multicolumn{2}{|c|}{ RVIN } & \multicolumn{2}{c|}{ SPN } \\
\cline { 2 - 5 } & $\mathbf{2 5 \%}$ & $\mathbf{5 0 \%}$ & $\mathbf{2 5 \%}$ & $\mathbf{5 0 \%}$ \\
\hline Median & 29.6031 & 22.4015 & 26.3844 & 15.4534 \\
\hline HF & $\mathbf{2 9 . 4 9 7 1}$ & $\mathbf{2 6 . 0 1 7 3}$ & 29.5311 & 23.3847 \\
\hline DBAIN & 15.7004 & 12.6862 & $\mathbf{3 5 . 0 1 8 5}$ & $\mathbf{2 9 . 4 5 4}$ \\
\hline
\end{tabular}

Figure 1: concordaerial image at varied impulse noise densities + Gaussian noise

$25 \% \mathrm{SPN}+$ gaussian

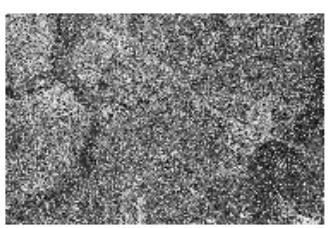

$50 \%$ spn+ Gaussian

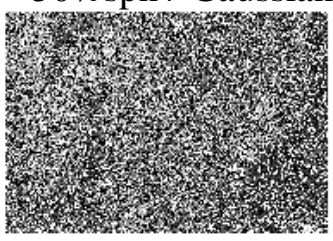

$25 \%$ RVIN +Gaussian hybrid filter

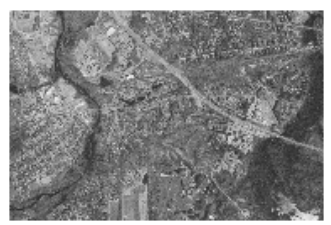

HF

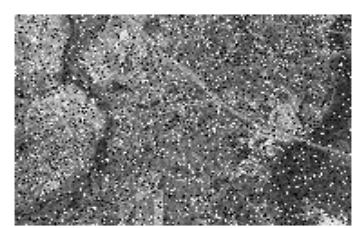

$\mathrm{HF}$

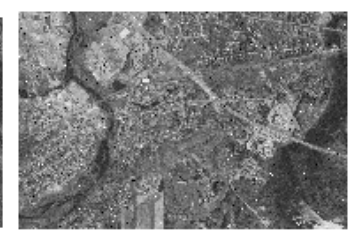

median

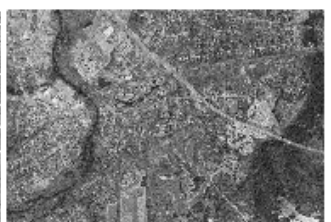

Median dba

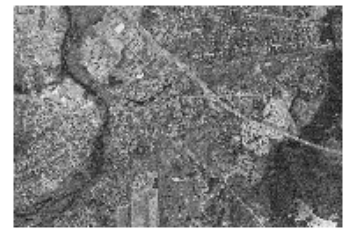

dba

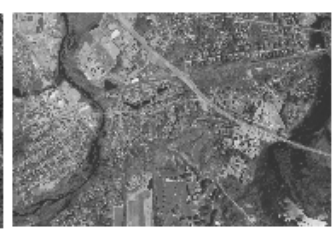

DBA 


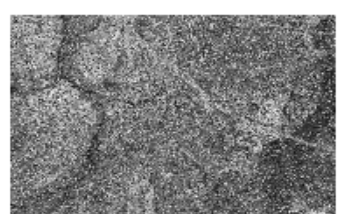

$50 \%$ rvin +gaussian

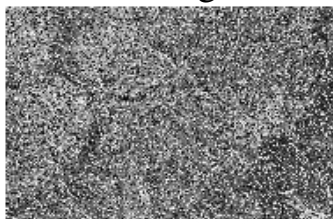

$25 \% \mathrm{SPN}$

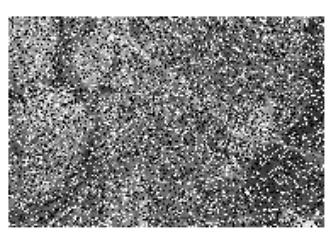

$50 \%$ spn

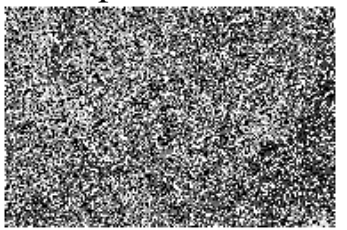

$25 \%$ rvin

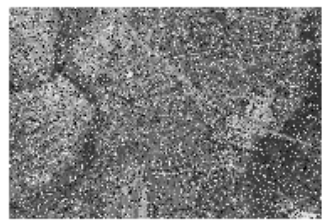

$50 \%$ rvin

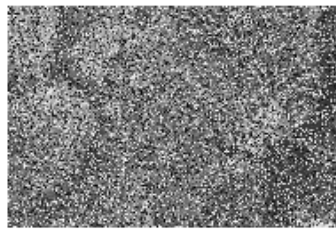

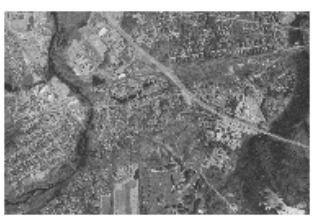

$\mathrm{HF}$

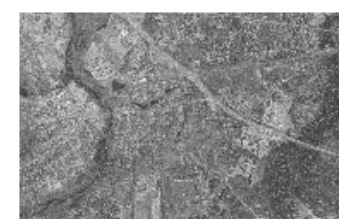

$\mathrm{HF}$

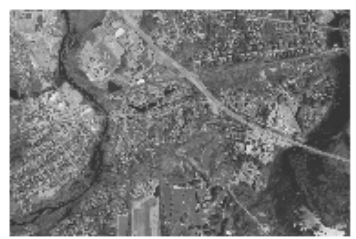

hf

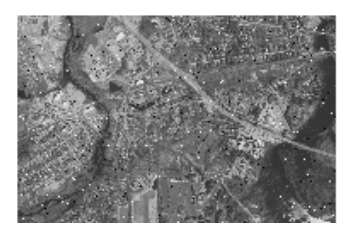

hf

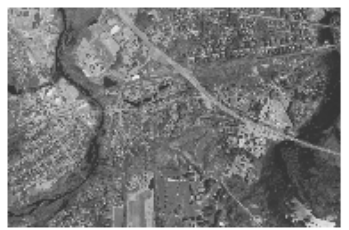

hf

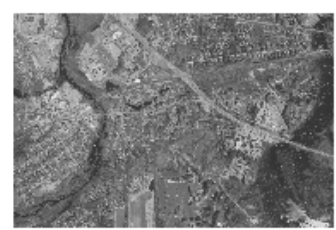

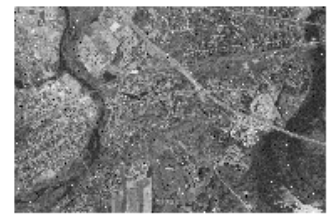

median

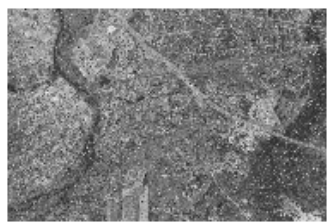

median

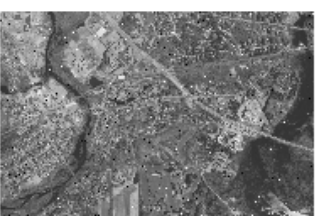

median

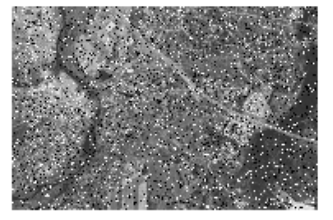

median

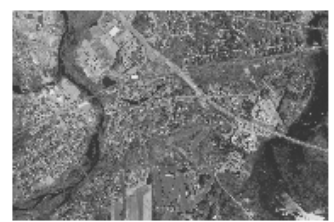

median

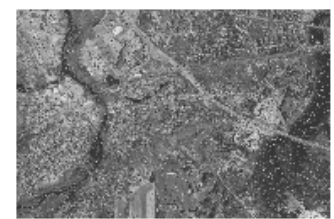

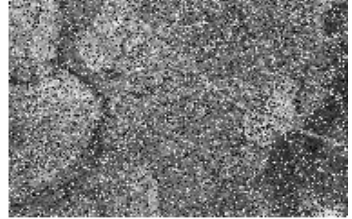

dba

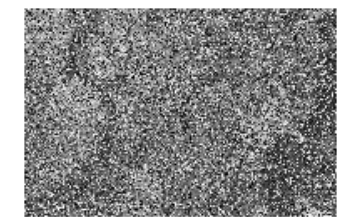

DBA

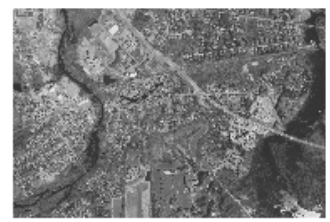

DBA

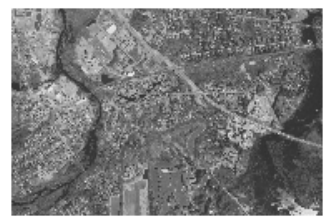

DBA

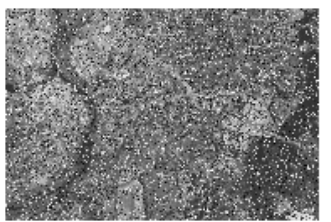

DBA

The noise distributions in different regions are different. It can be seen that in many method removes noise but generates false edges. The analysis shows that hybrid filter works better in the case of RVIN at both low and noise densities and RVIN +Gaussian noise. Decision based algorithm works better in the case of SPN and SPN+Gaussian at lower noise density.

\section{CONCLUSION}

In this paper variants of median filtering(order statistics) algorithms are applied on images to remove both SPN, RVIN and mixed noise. RS images when captured usually have Gaussian noise, speckle noise and salt and pepper noise and RVIN. In this work, three different image filtering algorithms of different schemes based on median filters are applied on the concordaerial.png. The performances of the filters are compared using the Peak Signal to Noise Ratio (PSNR). The analysis shows that hybrid filter works better in the case of RVIN and Gaussian + RVIN at both low and noise densities. Decision based algorithm works better in the case of SPN , Gaussian + SPN at both low and high noise densities. At low noise level AWGN 
Signal \& Image Processing : An International Journal(SIPIJ) Vol.1, No.2, December 2010

behaves like RVIN. Even if we apply wiener2 filter to remove the Gaussian noise the image reconstruction is only subtle improvement in mixed noise removal. The statistical and robustness properties of the (order statistics) median filter and its variants make them very suitable for impulse noise filtering.

\section{RFERENCES}

[1] H. Hwang and R. A. Haddad "Adaptive Median Filters: New Algorithms and Results" IEEE Transactions on image processing vol 4. P.no 499-502, Apr 1995.

[2] Aloke Datta "Removal of Random valued impulse noise"

[3] Jeny Rajan, K. Kannan and M.R. Kaimal, "An improved Hybrid Model for Molecular Image Denoising" Journal of Mathematical Imaging and Vision(2008)Vol.31 pp. 73-79.

[4] K.S.Srinivasan \& Ebenezer D "A New Fast and Efficient Decision-Based Algorithm for Removal of High- Density Impulse Noises", IEEE Sigmal Pricessing Letter, Vol. 14,No. 3, Mar 2007.

[5]. Ranganath R., Navalgund, V. Jayaraman and P. S. Roy "Remote sensing applications: An overview" Special Section: Indian Space Programme Current Science, Vol. 93, No. 12, 25 December 2007

[6] Order Statistics in Digital Image Processing ,Ioannis Pitas and Anastasios N. Venetsanopoulos, fellow, IEEE Proceedings of the ieee, vol. 80, no. 12, december 19921893

\section{Authors}

Mrs V.Radhika received her B.Sc (Phy) ,MCA and M.Phil degree from Bharathiar University in 1993 ,1996 and 2003 respectively. Currently she is pursuing her PhD at Avinashilingam university for women, Coimbatore. She is the Prof \& Head of the Department of Computer Science, in Sri Krishna Arts And Science College,Coimbatore. She has 14 years of teaching experience. Her areas of interest include Non-linear filtering techniques in Digital image processing.

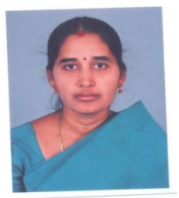

Dr. Padmavathi Ganapathi is the Professor and Head of the Department of Computer Science, Avinashilingam University for Women, Coimbatore. She has 21 years of teaching experience and one year Industrial experience. Her areas of interest include Network security and Cryptography and real time communication. She has more than 80 publications at national and International level. She is a life member of many professional organizations like CSI, ISTE, AACE, WSEAS, ISCA, and UWA. She is currently the Principal Investigator of 5 major projects under UGC and DRDO.

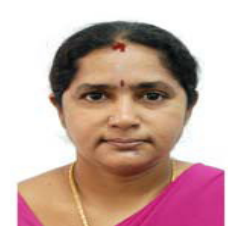

